\title{
Mitteilungen des Bundesverbands Geriatrie
}

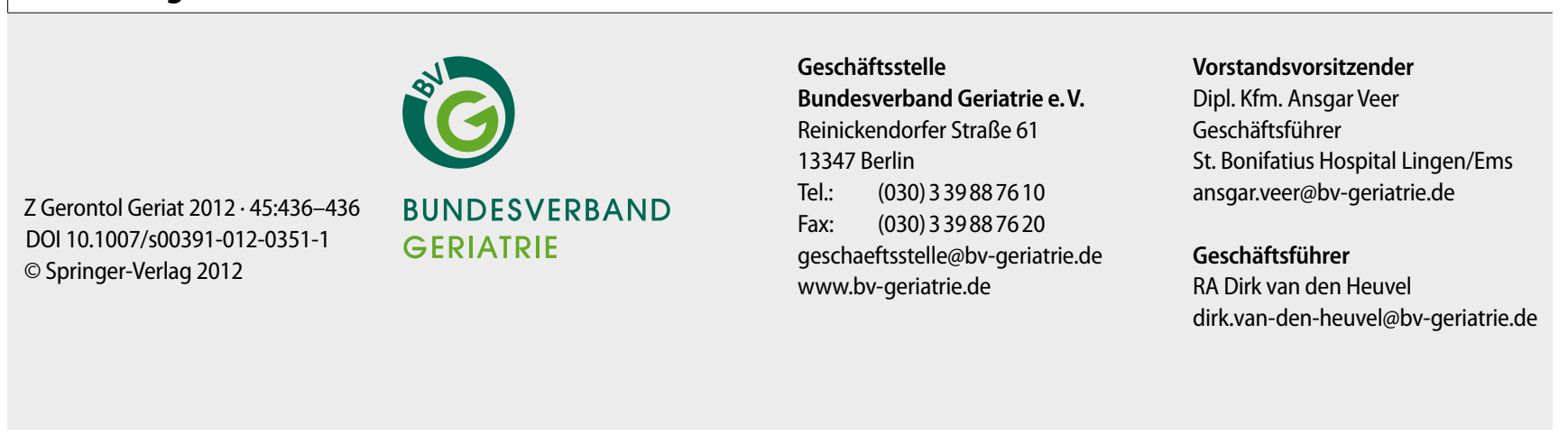

\section{Meldungen Juni/Juli 2012}

Sehr geehrte Damen und Herren, liebe Mitglieder,

genau am Tag des Redaktionsschlusses dieser Ausgabe ist der Bundesgesetzgeber noch einmal zum Themengebiet Geriatrie aktiv geworden. Der Bundestag hat im Rahmen der Verabschiedung des Psych-Entgelt-Gesetzes die gesetzliche Grundlage für die Einführung von geriatrischen Institutsambulanzen geschaffen. Damit wurde eine langjährige Forderung vom Bundestag aufgegriffen, die insbesondere vom Bundesverband immer wieder erhoben wurde. Die Verabschiedung im Bundestag ist jedoch nur der erste Schritt. In der Zeit zwischen Redaktionsschluss und Erscheinen dieser Ausgabe wird sich der Bundesrat noch mit dem Gesetz und damit auch mit der Regelung zu den geriatrischen Institutsambulanzen befassen müssen. Somit lässt sich zum heutigen Zeitpunkt noch keine abschließende Aussage dazu treffen, wie die gesetzlichen Regelungen letztlich genau aussehen werden.

Vor dem Hintergrund, dass in der Vergangenheit mehrere Bundesländer die Einführung dieser Institutsambulanzen sehr stark unterstützt haben - insbesondere das Ministerium in Sachsen hatte bereits mit einem konkreten $\mathrm{Ge}$ setzesvorschlag versucht, die geriatrischen Institutsambulanzen im Rahmen des zum 1. Januar dieses Jahres in Kraft getretenen GKV-Versorgungsstrukturgeset- zes zu verankern - hoffen wir, dass auch jetzt das Vorhaben grundsätzlich unterstützt wird. Seitens des Verbandes werden wir versuchen, die Beratungen im Rahmen des Bundesrates für weitere Verbesserungen des Gesetzestextes zu nutzen. Ein Anliegen des Verbandes ist es, auch Rehabilitationseinrichtungen sowie die im Verband vertretenen „Schwerpunktpraxen“ stärker einzubinden. Über die aktuelle Entwicklung werden wir Sie per Rundmail an die Mitglieder informieren.

Neben der "großen Politik“ rückt verbandspolitisch das Thema Messung bzw. Nachweis der Ergebnisqualität immer stärker in den Fokus der Aufmerksamkeit. In jüngster Zeit erreichen uns immer mehr Anfragen zu diesem Thema und immer öfter ist dieser Themenkomplex Gegenstand von Gesprächen mit Kostenträgern bzw. weiteren Beteiligten im Gesundheitssystem. Wir werden uns daher im Verband verstärkt um diesen Themenbereich kümmern, damit die Geriatrie in der Lage ist, die sich abzeichnenden Diskussionen fachlich-inhaltlich gut vorbereitet führen zu können.

So stand das Thema Ergebnisqualität unter anderem auch auf der Agenda eines Abstimmungsgespräches mit den beiden geriatrischen Fachgesellschaften (DGG und DGGG). Es ist gute Tradition, dass sich Vertreter aller drei Institutionen regelmäßig treffen und austauschen. Bei diesem
Treffen stand u.a. die inhaltliche Abstimmung zu dem Themenkomplex Alterstraumatologie im Mittelpunkt. So gibt es bereits nähere Überlegungen, Vorgaben für sogenannte Alterstraumatologische Zentren festzulegen.

Ein weiterer Punkt in dem Gespräch war die Zusammenarbeit von Gemidas Pro und GiB-Dat. Aktuell gibt es Gespräche zwischen dem Bundesverband und der $\mathrm{AfGiB}$, in denen Möglichkeiten einer stärkeren Kooperation zwischen beiden Datenbanksystemen erörtert werden. Derzeit wird abgeklärt, inwieweit die beiden Systeme inhaltlich und technisch kompatibel sind. Nach dieser Bestandsaufnahme können dann konkrete Fragen der Kooperation erörtert werden. Dabei sollen auch die weiteren in der Bundesrepublik bestehenden Datenbanksysteme mit eingebunden werden.

In den vergangenen Wochen hat der Bundesverband Geriatrie wieder mehrere Veranstaltungen für seine Mitglieder angeboten. Am 23. Mai fand unter anderem eine Veranstaltung zum Thema „Finanzierungsfragen in der Geriatrie" in Berlin statt. Nach einer gemeinsamen Einführung wurden in zwei Workshops die aktuellen Entwicklungen sowohl im akut-stationären wie auch im Reha-Bereich erörtert. Im Krankenhausbereich nahm das Thema PKMS-E einen breiten Raum ein, während im Reha-Workshop die Einführung der Schiedsstellen im Mittelpunkt stand. Neben der Information stand insbesondere der Austausch untereinander im Mittelpunkt der Veranstaltung.
Die Vortragsfolien können über die Homepage im Mitgliederbereich abgerufen werden.

Wie immer beginnt im Bundesverband im Sommer die inhaltliche Vorbereitung der Mitgliederversammlung im Herbst. Insofern möchte ich es nicht versäumen, Ihnen noch einmal unseren Tagungstermin zum Vormerken „ans Herz zu legen“. Die Mitgliederversammlung findet in diesem Jahr am 19. bzw. 20. November in Bayreuth statt. Die entsprechende Hotelliste ist bereits in einer der vorherigen Ausgaben dieser Zeitschrift veröffentlicht worden. Selbstverständlich können Sie diese Informationen auch noch einmal über die Geschäftsstelle anfordern.

Allen Mitgliedern eine schöne Sommer- und Urlaubszeit,

Dirk van den Heuvel Geschäftsführer 